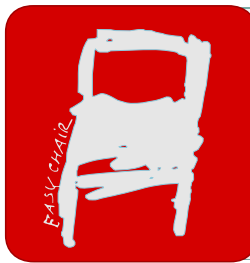

EPiC Series in Health Sciences

CAOS 2019. The 19th Annual Meeting of the International Society for Computer Assisted Orthopaedic Surgery

\title{
AMIS Total Hip Arthroplasty using traction table: 5 years follow-up
}

\author{
Thomas Apostolou ${ }^{1}$ and Ioanna P. Chatziprodromidou ${ }^{2}$ \\ ${ }^{1}$ School of Health Professionals, Alexander Technological Educational Institute of \\ Thessaloniki, Greece \\ ${ }^{2}$ Medical School, University of Patras, Greece \\ apostolouthomas@hotmail.com, ioannachatzi@med.upatras.gr
}

\begin{abstract}
Minimal invasive surgery has gained popularity among hip surgeons and patients. Based on early studies, the method is described as a very promising alternative, with low dislocation rates, resulting in a non-traumatic procedure and early functional return. However, complication rates arising of the recent studies raise concern about the applied technique. The aim of the study is to present the clinical results and intra- and post-operative complications of the AMIS procedure in patients with osteoarthritis of the hip, managed with total hip arthroplasty with positioning table, in a 5 years follow up. One senior hip arthroplasty surgeon performed all surgeries. Three hundred eighteen consecutive patients (195 females, 123 males) were clinically and radiologically evaluated, postoperatively 2, 6 and 12 months. Mean patient age was 69.7 years (24 to 88). There was significant improvement according to Harris-Hip Score, ODI, SF-36 scales. The mean incision length was $7.5 \mathrm{~cm}$ (6 to $8 \mathrm{~cm}$ ). The mean operating time was calculated at 85 minutes. The patients were discharged on the second post-operative day, able to walk with partial weight bearing. One month post-operative, the patients were advised for full weight bearing walking without crutches. Intraoperative complications included two femoral perforations. Postoperative complications included two patients with femoral fractures; one with dislocation; two with superficial infections; three with wound complications; three with femoral stem aseptic loosening; one with ceramic inner fracture and two acetabular component protrusion in the same patient, among which only the last patient had reoperation in both hips. Anterior Minimal Invasive Surgery of the hip is a non-traumatic procedure, associated with reduced pain, faster recovery and no major complications, but requires higher experience level from the hip surgeon.
\end{abstract}




\section{1ntroduction}

The direct anterior approach was initially described by Hueter in $1870^{1}$ and subsequently popularized by Smith-Petersen et al. ${ }^{2}$. Various surgical approaches have been applied over the last years for the total hip arthroplasty, but both surgeons and patients have demonstrated greater attention in the direct anterior approach, as it seems to offer rapid functional recovery of the patient, improved outcome in terms of hospitalization, muscle-sparing nature, pain and rehabilitation, and reduced risk of dislocation ${ }^{3}$. However, the steep learning curve, the difficulty of method application in obesity patients and the need for tendon and capsule further release remain some of the methods main disadvantages ${ }^{4}$.

Despite the recent surge in application of the direct anterior approach, clinical outcomes and complications of the newly launched AMIS method are sparsely published. The purpose of this study was to determine the method's early clinical outcomes with the use of traction table and demonstrate the intra- and post-operative complications.

\section{Materials and methods}

Three hundred eighteen primary total hip arthroplasties were included in our study. All operations were performed by a senior orthopaedic surgeon (TA) in a period between November 2013 to December 2018, using a direct anterior approach with a traction table. For almost 16 years, the orthopaedic surgeon used to perform the Harding procedure for all his hip arthroplasties cases. We identified 318 patients with a diagnosis of primary osteoarthritis of the hip (83\%), avascular necrosis of femoral head (7.6\%), ankylosing spondylitis (4.2\%), developmental hip dysplasia (1.8\%) and femoral neck fracture (3.4\%). Patients' mean age was 69.7 years (24 to 88).

The patient positioning, the operative field preparation, the skin incision, the intramuscular approach, the articular approach, the femoral neck cutting, the acetabular and femoral preparation, the reduction, the closure and all related procedures were performed as proposed by Laude ${ }^{5}$. The patient was placed in a supine position on a traction table (Medacta $\left.{ }^{\circledR}\right)$. All the following Medacta implants were used in all 318 cases: Versafit HA coated acetabular cup, ceramic inner, Quadra porous coated stem, ceramic femoral head $(32 \mathrm{~mm}$ diameter for $48 \mathrm{~mm}$ acetabular cup and $36 \mathrm{~mm}$ diameter for greater acetabular cup sizes). Screws were used in $87 \%$ of the cases for primary stability of the acetabular cup. No fluoroscopy was used intraoperatively.

One single orthopaedic surgeon (TA) performed all clinical and radiographic evaluations of the patients at 2, 6 and 12 months post-op.

Harris-Hip Score (HHS) was used for all 318 cases. Statistical analysis was carried out by SPSS software (Statistical Package for the Social Sciences, version 24).

\section{Results}

The mean HHS was calculated 82. The mean incision length was measured at $7,5 \mathrm{~cm}$. The operating time was recorded at 85 minutes. All patients were discharged on the 2nd post-op day, with partial weight bearing and were indicated for full weight bearing walking without any support, 1 month post-op. There were $2(0.006 \%)$ intra-operative complications, namely femoral perforations, which both occurred during the preparation of the femoral bed with the wrasp tool application. There were $14(0.04 \%)$ postoperative complications, which included: i) two female patients who sustained femoral fractures 6 and 12 months post-op, after a fall in their house. Both were treated conservatively and hip fractures were healed 3 months later, ii) one patient with posterior dislocation of the hip at the 
2nd day post-operatively, after he was discharged from the hospital and in an attempt to enter to the car to be transferred to his home. Closed reduction was performed under anesthesia and after one month of bed rest, the patient was successfully mobilized with no further complications, iii) five patients with superficial wound infections, that were treated with antibiotics per os. There was no need of further hospitalization, iv) three middle-aged active male patients with femoral stem aseptic loosening, 3, 6 and 11 months post-operatively. Revision of the femoral stem was performed using bigger size primary stems, v) one patient with ceramic inner fracture. Misplacement of the ceramic inner was identified in the 2nd month's follow-up evaluation and resulted in ceramic inner fracture 12 months post operatively. Revision of ceramic inner and femoral head was performed and vi) two cases with acetabular component protrusion, which both occurred in the same patient. A female patient (41 years old) with developmental dysplasia of the hip and acetabular component protrusion, 1 day post-op and the revision reinforcement COMBI cup was implanted. The same complication was recorded after her second hip replacement, 5 months post operatively and the revision of the acetabular was performed with the same reinforcement acetabular implant. All the revisions were performed with the same procedure, with no extension of the primary incision.

\section{Discussion}

In this study, we investigated all hip arthroplasties dealt in our clinic with direct anterior approach and presented various outcome measures and complications met. Seng et al. ${ }^{6}$ assessed post-operative recovery using HHS at 81 , almost similar to ours. Concerning incision length, Šebečić et al. ${ }^{7}$ and Sendtner et al. ${ }^{8}$ recorded mean incision lengths of 7,5 and $8,5 \mathrm{~cm}$, respectively, confronting to our findings. Berend et al. ${ }^{9}$ recorded that their operating time was 69 minutes, significantly lower than ours. As in our study, the operating time was significantly higher during the first 20 cases but dropped during the study, and this was attributed to the surgeon's learning curve. Although literature insists that hospitalization length of stay is not significantly associated, the mean length of stay was between 2 to 5 days in most studies ${ }^{10}$. De Geest et al. ${ }^{11}$ conducted a study similar to ours and concerning complications, they had 9(3\%) intra-operative complications (trochanter avulsion fractures, fractures of the proximal calcar and femoral perforations) and 42 (14\%) post-operative complications (periprosthetic femur fractures and infection).

This study was not without limitation. The number of cases included, the follow-up format applied, the subjectivity of patients' clinical and radiological evaluation may be considered as mail limitations of this study.

In conclusion, current evidence justifies recent enthusiasm for AMIS technique but has a considerable learning curve, which mainly affects its outcomes and determines its complicati

\section{References}

1. Hueter C. Grundriss Der Chirurgie. FCW Vogel; 1883.

2. Smith-Petersen MN, LARSON CB, AUFRANC OE, LAW WA. COMPLICATIONS OF OLD FRACTURES OF THE NECK OF THE FEMUR RESULTS OF TREATMENT BY VITALLIUM-MOLD ARTHROPLASTY. JBJS. 1947;29(1):41-46.

3. Kyriakopoulos G, Poultsides L, Christofilopoulos P. Total hip arthroplasty through an anterior approach: the pros and cons. EFORT open Rev. 2018;3(11):574-583. 
AMIS Total Hip Arthroplasty using traction table: 5 years ... T. Apostolou and I. Chatziprodromidou

4. Barrett WP, Turner SE, Leopold JP. Prospective randomized study of direct anterior vs postero-lateral approach for total hip arthroplasty. J Arthroplasty. 2013;28(9):1634-1638.

5. Laude F. Total hip arthroplasty through an anterior Hueter minimally invasive approach. Interact Surg. 2006;1(1-4):5-11.

6. Seng BE, Berend KR, Ajluni AF, Lombardi Jr A V. Anterior-supine minimally invasive total hip arthroplasty: defining the learning curve. Orthop Clin North Am. 2009;40(3):343-350.

7. Šebečić B, Starešinić M, Čuljak V, Japjec M. Minimally invasive hip arthroplasty: advantages and disadvantages. Med Glas. 2012;9(1).

8. Sendtner E, Borowiak K, Schuster T, Woerner M, Grifka J, Renkawitz T. Tackling the learning curve: comparison between the anterior, minimally invasive (Micro-hip ${ }^{\circledR}$ ) and the lateral, transgluteal (Bauer) approach for primary total hip replacement. Arch Orthop Trauma Surg. 2011;131(5):597-602.

9. Berend KR, Lombardi Jr A V, Seng BE, Adams JB. Enhanced early outcomes with the anterior supine intermuscular approach in primary total hip arthroplasty. JBJS. 2009;91(Supplement_6):107-120.

10. Meermans G, Konan S, Das R, Volpin A, Haddad FS. The direct anterior approach in total hip arthroplasty: a systematic review of the literature. Bone Joint J. 2017;99(6):732-740.

11. De Geest T, Vansintjan P, De Loore G. Direct anterior total hip arthroplasty: Complications and early outcome in a series of 300 cases. Acta Orthop Belg. 2013;79(2):166-173. 